\title{
An Adaptive Frequency and Time Hopping PPM UWB for Multiple Access Communication
}

\author{
G. S. Biradar, S. N. Merchant, U. B. Desai \\ SPANN Laboratory, Department of Electrical Engineering \\ Indian Institute of Technology Bombay, Mumbai, INDIA-400076 \\ email:\{gsbiradar, merchant, ubdesai\}@ee.iitb.ac.in
}

\begin{abstract}
Typical Ultra Wide Band (UWB) systems are built to meet Quality of service $(\mathrm{QoS})$ constraints under multiple access environment. We propose a UWB physical layer that adapts its number of frequency and time hops to efficiently meet QoS requirements. The proposed adaptive UWB uses side information to know the current QoS and adjusts its number of frequency and time hops accordingly. The system employs adaptive frequency and time hopping pulse position modulation (AFTH-PPM). We have derived an expression for the bit error probability for single user and multiple user synchronous transmitter case under additive white Gaussian noise (AWGN). The bit error rate (BER) performance is evaluated under AWGN with multiple access communication.
\end{abstract}

Keywords- Adaptive systems, Frequency hopping, Modulation, Multiple access, Time hopping, UWB.

\section{INTRODUCTION}

Ultra wide band [1], [2] is a new technology that has the potential to revolutionize wireless communication by delivering high data rates with very low power densities. Since they use very short pulses with approximately one nano second, the signal energy is widely spread up to several $\mathrm{GHz}$, which may interfere with existing narrowband communication systems. In order to avoid interference to existing narrowband systems we must strictly meet the FCC rules for power limitation. This restricts the use of UWB for personal area networks (PAN).

Under multiple access scenario the presence of multiple user signals transmitting at same time is typical source of interference for wireless signals. There are several multiple access schemes namely Time Hopping (TH) [3], [4], Frequency Hopping (FH) [5] and Direct Sequence (DS) [6] wherein orthogonal codes are used to avoid multiple access interference (MAI). Efforts have been made to reduce the multiple access interference by designing orthogonal hopping sequences [7]. However in practice, the received signal from different users are not orthogonal because of multipath and asynchronous transmission and varying number of UWB transmitters. And also it is not possible to design orthogonal codes for all shifts. Because of varying number of active UWB devices MAI also varies. In order to meet QoS UWB has to adapts its parameters so that MAI is avoided. Reference [8] proposed adaptive Mary PPM modulation for optimization interms of data rate and energy to meet QoS requirements. It is possible to meet the required QoS using only adaptive time hopping but at the cost of reduced data rate.
In this paper, we propose a new scheme adaptive frequency and time hopping, which adapts its number of frequency and time hops depending on the required bit error rate (BER) in multiple access communication under additive white Gaussian noise (AWGN). This new technique overcomes the problem suffered by adaptive time hopping. The exact probability of error is derived for single user and multiple user under synchronous transmitter case. Simulation is carried out for both synchronous and asynchronous cases. Second derivative of Gaussian pulse is considered for multiple access analysis.

The paper is organized as follows. In Section II, the system model and construction of the frequency and time hopping PPM UWB signals is described. In Section III error probability of M-ary PPM over AWGN channel is presented. In Section IV multiple access interference and error probability analysis is presented. In Section V adaptive frequency and time hopping UWB explained. Simulation results and performance is given in Section VI. Finally Section VII provides conclusion.

\section{SIGNAL AND SYSTEM MODEL}

The frequency and time hopping M-ary PPM system model for $v$ th user in given by

$s^{v}(t)=\sum_{j=-\infty}^{\infty} A_{d_{\left\lfloor j / N_{s}\right\rfloor}}^{(v)} p\left(t-j T_{f}-c_{j}^{(v)} T_{c}-\delta_{d_{\left\lfloor j / N_{s}\right\rfloor}^{(v)}}\right) e^{-j 2 \pi k_{j}^{(v)} t}$

where $A^{(v)}$ is the signal amplitude, $p(t)$ represents the second derivative of Gaussian pulse with pulse width $T_{p}, T_{f}$ is the frame time, frame is divided into $N_{t h}$ time slots with duration $T_{c}$. The pulse shift pattern $c_{j}^{(v)}, 0 \leq c_{j}^{(v)} \leq N_{t h}$ $\left(N_{t h} * T_{c}=T_{f}\right)$ also called the time hopping sequence for $v$ th source and it is pseudo random with period $T_{c}$, this additional shift avoids catastrophic collisions due to multiple access interference. The sequence $d$ is the data stream generated by the $v$ th source after channel coding and $\delta$ is the additional time shift utilized by M-ary PPM. $N_{s}$, represents repetition code length, that is, $N_{s}$ pulses are used to transmit the same information. Frequency spectrum is divided into $N_{f h}$ bands with minimum bandwidth of $500 \mathrm{MHz} . k_{j}^{(v)}$ is carrier frequency during the $j$ th frame of $v$ th user which is pseudo random and takes any one of the frequency band $0 \leq k_{j}^{(v)} \leq N_{f h}\left(N_{f h} * B_{f}=B\right)$. Fig. 1 shows the frequency time hopping presentation of the 


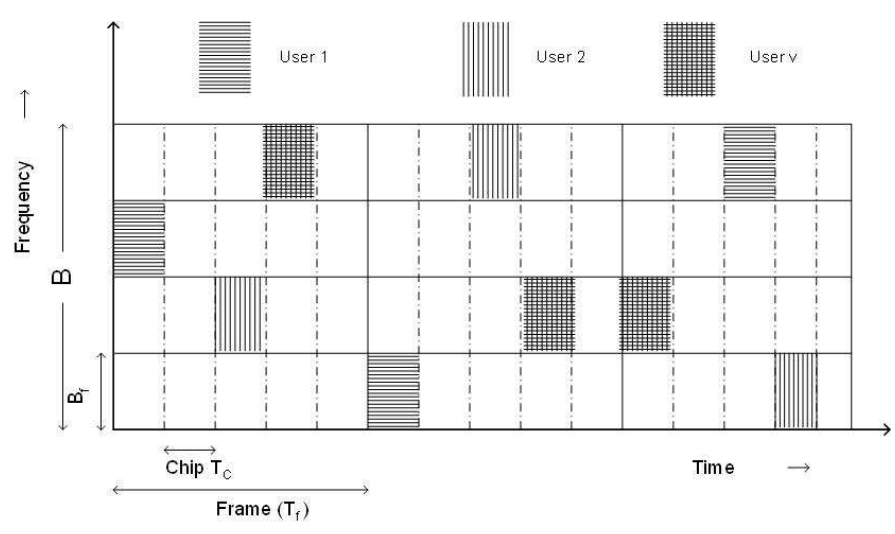

Fig. 1. Principle of frequency and time hopping UWB system

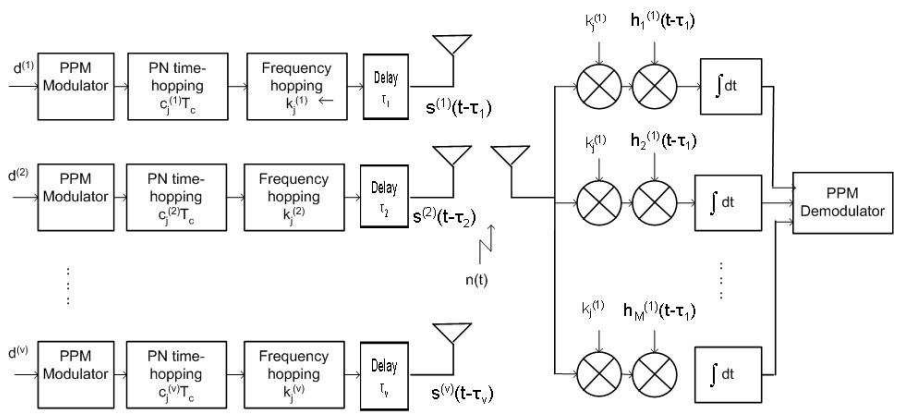

Fig. 2. System model of FTH-MA M-ary PPM UWB system

UWB signals for multiple users. Here the UWB pulse is transmitted in any one time slot occupying $T_{c}$ seconds and $B_{f}$ band width

For M-ary PPM, signal amplitude $A^{(v)}=1$ so that (1) can be written as

$$
s^{v}(t)=\sum_{j=-\infty}^{\infty} p\left(t-j T_{f}-c_{j}^{(v)} T_{c}-\delta_{d_{\left\lfloor j / N_{s}\right\rfloor}^{(v)}}\right) e^{-j 2 \pi k_{j}^{(v)} t}
$$

The received signal after frequency demodulation can be modeled as:

$$
\begin{gathered}
r(t)=\sum_{v=1}^{N_{u}}\left(s^{(v)}\left(t-\tau_{v}\right)\right)+n(t) \\
r(t)=\sum_{v=1}^{N_{u}} \sum_{j=-\infty}^{\infty} p\left(t-j T_{f}-c_{j}^{(v)} T_{c}-\delta_{d_{\left\lfloor j / N_{s}\right\rfloor}^{(v)}}\right)+n(t)
\end{gathered}
$$

where $n(t)$ is AWGN noise with power spectral density $N_{0} / 2, \tau_{v}$ is the propagation delay for the $v$ th user, $p(t)$ is the received pulse waveform. PPM receiver uses bank of M correlation receivers followed by a detector. Even though the number of users are more than one still an M-ary correlation receiver is typically used for simplicity.

\section{ERROR PROBABILITY OF M-ARY PPM OVER AWGN CHANNEL}

The vector representation of an M-ary PPM for single user case is defined as an $\mathrm{P}$ dimensional vector with nonzero value in $m$ th dimension.

$$
\boldsymbol{s}_{m}=\left[0, \ldots, \sqrt{E_{g}}, 0 \ldots .0\right]
$$

where $\sqrt{E}_{g}$ is the average signal energy.

The received signal can be expressed as

$$
r=s+n
$$

As illustrated in Fig. 2 optimal receiver for M-ary orthogonal PPM signals consists of a parallel bank of $\mathrm{M}$ cross correlators. Let $h_{j}, 1 \leq j \leq M$, denote the $j$ th basis signal vector, which is the vector representation of the basis function $h_{j}(t)$ shown in fig. 1, defined as

$$
h_{j}=[0, \ldots ., 0,1, \ldots ., 0]
$$

where the non zero value 1 is in the $j$ th dimension. Assuming $s_{m}$ was sent, the optimum detector makes a decision on $s_{m}$ in favor of the signal corresponding to the cross correlator with the minimum Euclidian distance.

$$
C\left(\boldsymbol{r}, \boldsymbol{h}_{j}\right)=\boldsymbol{r} \cdot \boldsymbol{h}_{j} \quad j=1,2, \ldots, M
$$

where

$$
\begin{gathered}
C\left(\boldsymbol{r}, \boldsymbol{h}_{j}\right)=n_{j} \quad j \neq m \\
C\left(\boldsymbol{r}, \boldsymbol{h}_{m}\right)=\sqrt{E_{g}}+n_{m}
\end{gathered}
$$

Considering $N s=1$, the demodulated signal $\hat{\boldsymbol{s}}$ is given by

$$
\hat{\boldsymbol{s}}=\arg \min _{\boldsymbol{s}_{j}}\left\|C\left(\boldsymbol{r}, \boldsymbol{h}_{j}\right)-\sqrt{E_{g}}\right\| \quad j=1,2, \ldots ., M
$$
[11]

The average probability of a correct decision is given by

$$
p_{c}=\int_{-\infty}^{\infty}\left(\frac{1}{\sqrt{2 \pi}} \int_{-\infty}^{\boldsymbol{r} / \sqrt{N_{0} / 2}} e^{-x^{2} / 2} d x\right)^{M-1} p(\boldsymbol{r}) d \boldsymbol{r}
$$

where,

$$
p(\boldsymbol{r})=\frac{1}{\sqrt{\pi N_{0}}} \exp \left(\frac{-\left(\boldsymbol{r}-\sqrt{E_{g}}\right)^{2}}{N_{0}}\right)
$$
is

Finally, the probability of a symbol error for an M-ary PPM

$$
P_{M}=1-P_{c}
$$




\section{Multiple ACCESS INTERFERENCE AND ERROR PROBABILITY}

MAI is the factor limiting the performance and capacity of the system when more than one user is active. MAI can be modeled as a zero mean Gaussian random variable if number of users are large [9]. Assuming M-ary PPM signal are orthogonal (i.e $\delta \geq T_{p}$ ) the MAI and error probability analysis given in section III for single user system can be extended to multiple access system.

\section{A. Multiple access interference and error probability}

MAI is the factor limiting the performance and capacity of the system when more than one user is active. MAI can be modeled as a zero mean Gaussian random variable if number of users are large [9]. Assuming M-ary PPM signal are orthogonal (i.e $\delta \geq T_{p}$ ) the MAI and error probability analysis given in section III for single user system can be extended to multiple access system.

\section{B. Multiple access interference and error probability}

As given in (3) the base band received signal is modeled as

$$
r(t)=\sum_{v=1}^{N_{u}}\left(s^{(v)}\left(t-\tau_{v}\right)\right)+n(t)
$$

to evaluate the MAI, we make the following assumptions:

(a) $s^{(v)}\left(t-\tau_{v}\right)$ for $v=1,2, \ldots, N_{u}$, where $N_{u}$ is the number of active users, and the noise $n(t)$ is assumed to be independent.

(b) The time hopping sequence $c_{j}^{(v)}$ and time delay $\tau_{v}$ are assumed to be independent and identically distributed (iid) over the time interval $\left[0, T_{f}\right]$.

(c) The frequency hopping sequence $k_{j}^{(v)}$ is assumed to be independent and identically distributed over the frequency band $B$.

(d) Perfect synchronization is assumed at the receiver that is $\tau_{v}$ is known at the receiver.

Assuming that $N_{s}=1$ and desired user corresponds to $v=1$.

The M-ary correlation receiver for user 1 consists of $\mathbf{M}$ cross correlators with basis function $h_{i}^{(1)}(t)$

$$
h_{i}^{(1)}(t)=p\left(t-\delta_{i}-\tau_{1}\right), \quad i=1,2, \ldots, M .
$$

At sample time $t=j T_{f}$, the output of each filter $\hat{\boldsymbol{r}}_{i}, i=$ $1, \ldots . ., M$ is

$$
\begin{gathered}
\hat{\boldsymbol{r}}_{i}=\sum_{j=n N_{s}+1}^{(n+1) N_{s}} \int_{(j-1) T_{f}}^{T_{f}} \boldsymbol{r}(t) h_{i}^{(1)}\left(t-j T_{f}-c_{i}^{(v)} T_{c}-\delta_{i}\right) d t \\
i=1,2, \ldots, M .
\end{gathered}
$$

Assuming PPM signal $\boldsymbol{s}_{m}$ is transmitted by user 1, (17) can be written as

$$
\hat{\boldsymbol{r}}_{i}= \begin{cases}N_{s} \sqrt{E_{g}}+N_{M A I}+N & i=n \\ N_{M A I}+N & i \neq n\end{cases}
$$

where MAI component $N_{M A I}$ is

$N_{M A I}=\left\{\begin{array}{lc}\sum_{v=2}^{N_{u}} \sum_{j=n N_{s}+1}^{(n+1) N_{s}} \int_{(j-1) T_{f}}^{T_{f}} p\left(t-j T_{f}-c_{j}^{(v)} T_{c}-\delta_{d_{\left\lfloor j / N_{s}\right\rfloor}^{(v)}}\right) \\ \times p\left(t-\delta_{i}-\tau_{1}-j T_{f}-c_{j}^{(v)} T_{c}\right) d t & k_{j}^{(1)}=k_{j}^{(v)} \\ 0 & \end{array}\right.$

and AWGN component $\mathrm{N}$ is

$N=\sum_{j=n N_{s}+1}^{(n+1) N_{s}} \int_{(j-1) T_{f}}^{T_{f}} n(t) p\left(t-\delta_{i}-\tau_{1}-j T_{f}-c_{j}^{(v)} T_{c}\right) d t$

By defining the autocorrelation function of $p(t)$ as

$$
\rho(\Delta)=\int_{0}^{T_{f}} p(t) p(t+\Delta) d t
$$

(19) can be written as

$$
N_{M A I}= \begin{cases}\sum_{j=1}^{N_{s}} \sum_{v=2}^{N_{u}} \rho\left(\Delta_{j}^{(v)}\right) & k_{j}^{(1)}=k_{j}^{(v)} \\ 0 & \text { otherwise }\end{cases}
$$

where $\Delta_{j}^{(v)}=\left(c_{j}^{(1)}-c_{j}^{(v)}\right) T_{c}-\left(\delta_{i}^{(1)}-\delta_{d_{\lfloor j / N,\rfloor}^{(v)}}\right)-\left(\tau_{1}-\tau_{v}\right)$ is the time difference between user 1 and user $v$.

Under the assumptions listed above, $\Delta$ can be modeled as a random variables uniformly distributed over $\left[-T_{f}, T_{f}\right]$. The MAI is modeled as a Gaussian random process for the multi-user environment [10]. With the Gaussian approximation we require the mean and variance of (18) to characterize the output of the cross correlators.

The AWGN component has zero mean and variance $N_{s} N_{0} / 2$, the mean and variance of MAI are pulse waveform specific. The calculations are carried out considering double differentiated Gaussian pulse as the transmitted pulse and all PPM signals are equally likely apriori, The double differentiated Gaussian pulse is defined as

$$
p(t)= \begin{cases}\sqrt{E_{g}}\left(1-\frac{4 \pi t^{2}}{\lambda T_{p}^{2}}\right) e^{-\frac{2 \pi t^{2}}{\lambda T_{p}^{2}}} & \frac{-T_{p}}{2} \leq t \leq \frac{T_{p}}{2} \\ 0 & \text { otherwise }\end{cases}
$$


where, $\lambda$ is pulse shaping parameter, The autocorrelation of double differentiated Gaussian pulse is then

$\rho(\Delta)=\left\{\begin{array}{l}E_{g} \sqrt{\frac{\lambda T_{p}^{2}}{8}} e^{-\frac{\pi \Delta^{2}}{\lambda T_{p}^{2}}}(1.0606- \\ \left.30.72 \frac{\Delta^{2}}{\lambda T_{p}^{2}}+11.6856 \frac{\Delta^{4}}{\lambda^{2} T_{p}^{4}}\right) \\ 0\end{array}\right.$

$0 \leq|\Delta| \leq \frac{T_{p}}{2}$ otherwise

considering $\lambda=1$

$$
\begin{gathered}
E[M A I]=0 \\
\operatorname{Var}\left[N_{M A I}\right]=N_{s} E_{g}\left(N_{u}-1\right) \frac{35 \sqrt{2} T_{p}}{2 * 96 T_{f}} \\
\operatorname{Var}\left[N_{M A I}\right] \approx N_{s} E_{g}\left(N_{u}-1\right) \frac{T_{p}}{4 T_{f}}
\end{gathered}
$$

since $T_{f} / 2 T_{p}=N_{t h}(26)$ can be written as,

$$
\operatorname{Var}\left[N_{M A I}\right] \approx N_{s} E_{g}\left(N_{u}-1\right) \frac{1}{8 N_{t h}}
$$

Now because of frequency hopping it can be easily shown that $\operatorname{Var}\left[N_{M A I}\right]$ will be reduced by a factor $N_{f h}$ and (27) can be rewritten as

$$
\operatorname{Var}\left[N_{M A I}\right] \approx N_{s} E_{g}\left(N_{u}-1\right) \frac{1}{8 N_{t h} N_{f h}}
$$

Note that $\sigma_{M A I}^{2}$ increases with $N_{s}, E_{g}$ and the number of users $N_{u}$, but decreases with the spread ratio $N_{t h}$ and $N_{f h}$

Using standard techniques [11] the average probability of error for a single user under multiple access interference for binary PPM is given by

$$
P_{M} \leq Q\left(N_{s} \sqrt{\frac{E_{g}}{\sigma_{M A I}^{2}+\frac{N_{s} N_{0}}{2}}}\right)
$$

\section{AdAPtive Frequency AND Time Hopping UWB}

Under the multiple access environment traditional communication with fixed modulation scheme is inadequate to efficiently meet QoS requirements. In (28) it is shown that MAI can be reduced by increasing the number of frequency hops or time hops. Hence to meet QoS requirements we propose dynamically changing number of frequency and time hops.

In adaptive frequency and time hopping-UWB the first step is to examine the current QoS, the second step is to check the required QoS, the third step is to adapt number of hops accordingly. In this paper QoS is BER and data rate. Since the communication among UWB is not centrally controlled the number of active UWB devices in the area is not known. In order to know current QoS we propose two adaptation schemes:

(i) UWB trans-receiver transmits bits at regular intervals and listens to calculate the BER. Depending on this information number of hops are varied to meet required BER.

(ii) All active UWB devices regularly send "Hello" messages

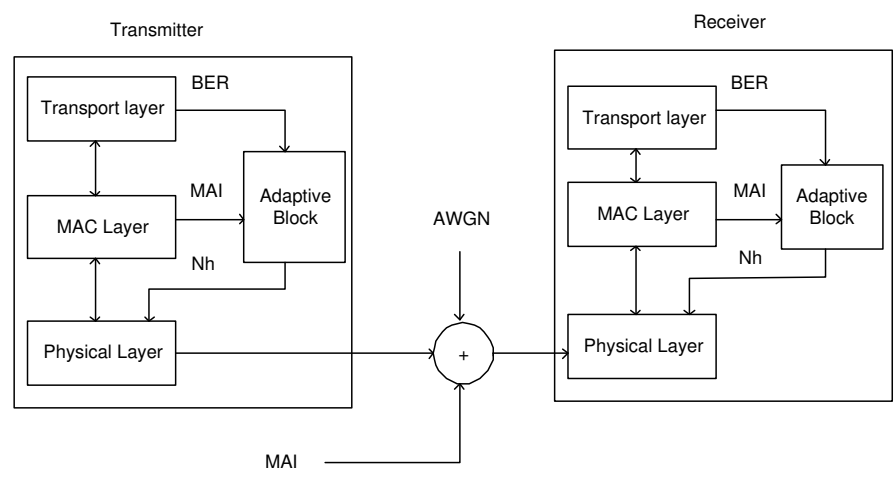

Fig. 3. Block diagram of adaptive UWB system

so that number of active UWB devices in the area is known to each other. Depending on this information number of hops are varied to meet the required BER.

\section{Simulations AND RESUlts}

Simulations were carried out for both synchronous and asynchronous user cases. Bit error rate results are presented as a function of $E_{b} / N_{0}$ and number of users. The parameters considered for simulations are binary PPM with sampling frequency of $50 \mathrm{MHz}$, chip time of 1 nanosecond, double differentiated Gaussian pulse of width 0.5 nanosecond and $\delta$ of 0.5 nanosecond. Frequency spectrum $3.1 \mathrm{GHz}-10.6 \mathrm{GHz}$ is divided into maximum of 8 bands $\left(N_{f h}\right)$ each of $900 \mathrm{MHz}$ with a guard band of $42 \mathrm{MHz}$. Pseudo random frequency and time hopping codes of length 50000 is generated and assigned to each user.

Binary data is generated using uniform random number generator for each user and modulated using UWB pulse. AWGN noise is generated and added to modulated signal.

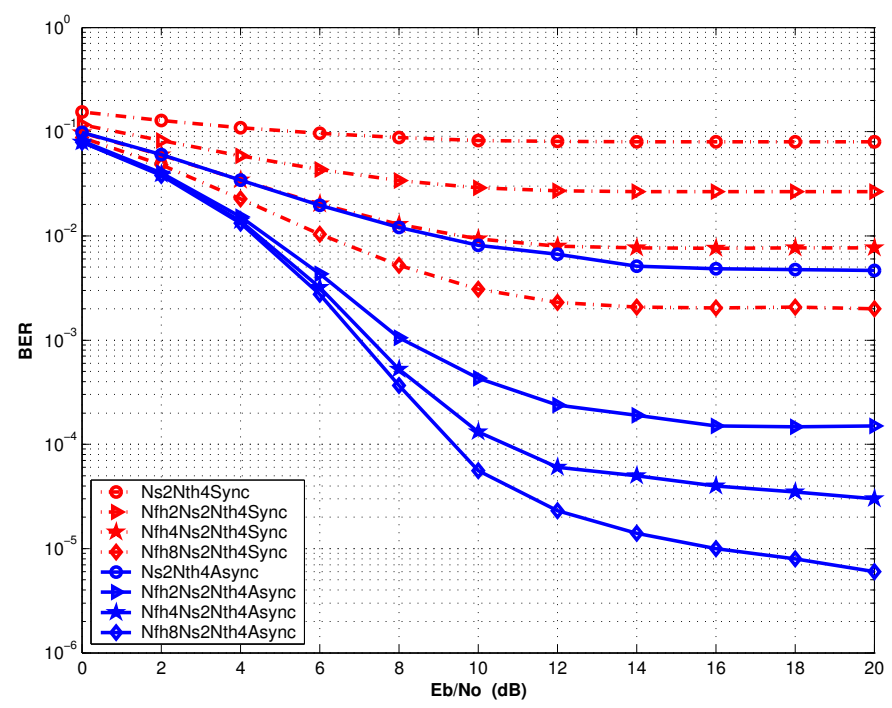

Fig. 4. BER plot for $N_{o u}=4, N_{s}=2, N_{t h}=4, N_{f h}=2,4,8$ 


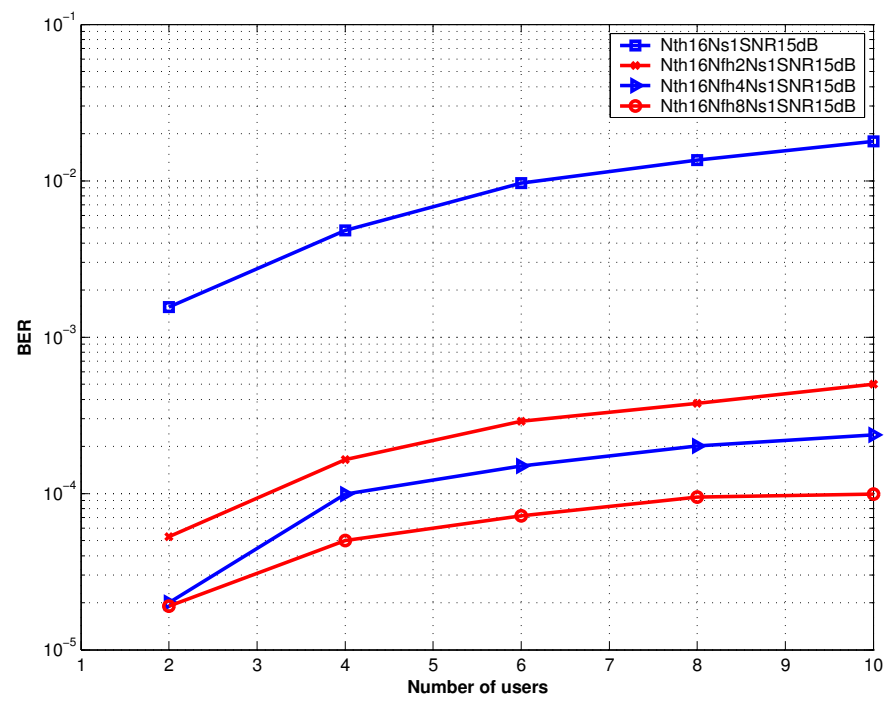

Fig. 5. Plot for Number of users vs BER

Simulations are carried out for fixed number of time hops $\left(N_{t h}\right)$ and different frequency hops $\left(N_{f h}\right)$ and repetitive coding $\left(N_{s}\right)$. Data of length $10^{6}$ bits is transmitted and BER performance tested. Receiver uses a frequency demodulator followed by correlation type detector, it is assumed that frequency and time hopping sequence of user of interest is known.

Fig. 4 shows BER plot for time hopping and frequency-time hopping for the cases of 4 synchronous and asynchronous users with $N_{s}=2, N_{t h}=4$ and variable $N_{f h}=2,4$ and 8 . It is observed that introduction of frequency hopping along with time hopping greatly reduces MAI and hence reduction in bit error probability. further doubling the frequency hops improves BER performance by $2 \mathrm{~dB}$.

It is also observed that asynchronous transmission performance is better than synchronous transmitter case. This is because of the probability of two or more users transmitting

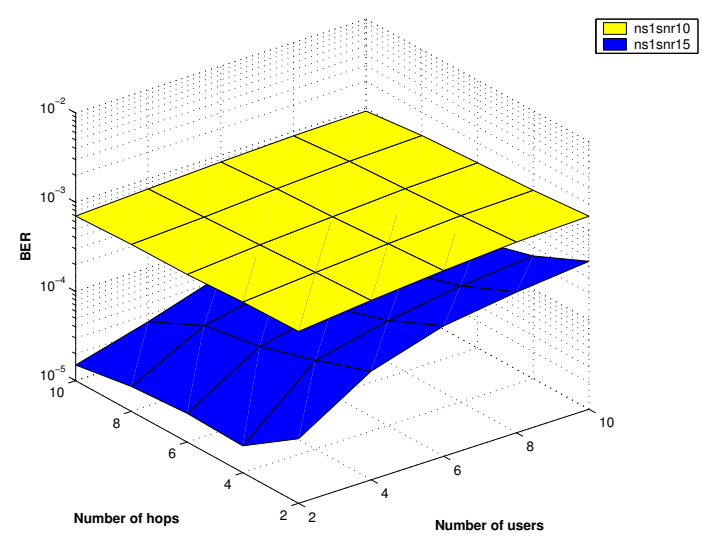

Fig. 6. Plot for Number of users, no of hops vs BER simultaneously is low which results in less interference and consequently BER performance improves.

Fig. 5 shows plot of number of users versus probability of error for just time hopping and frequency-time hopping asynchronous transmitters with $N_{t h}=16 N_{f h}=2,4$ and 8 for $E_{b} / N_{0}$ of $15 \mathrm{~dB} N_{s}=1$. It can be observed that probability of error decreases with introduction of frequency hopping and improves by $2 \mathrm{~dB}$ for every double in frequency hopping.

Fig. 6 shows BER plot as a function of number of users and number of frequency hops for a fixed $N_{t h}=16$ for SNR of $10 \mathrm{db}$ and $15 \mathrm{~dB}$ with $N_{s}=1$.

Therefore using these plots the system can adapt different frequency and time hops for the required BER under multiple access condition.

\section{CONCLUSION}

In this paper we proposed an adaptive frequency and time hopping PPM for UWB to maintain the required BER performance under AWGN and multiple access environment. It is shown that significant improvement is achieved as the number of hops are increased, Based on the exhaustive simulation results we conclude that the value of $N_{f h} * N_{t h}$ which is 12 times the number of users is sufficient to maintain a BER of $10^{-4}$ with $E_{b} / N_{0}$ of $10 \mathrm{~dB}$.

\section{REFERENCES}

[1] R.A. Scholtz, "Multiple access with time-hopping impulse modulation," in Proc. IEEE Military Communications Conference (MILCOM'93), vol. 2, pp. 447-450, Boston, Mass, USA, Oct. 1993.

[2] F. Ramirez-Mireles and R.A. Scholtz, "System performance analysis of impulse radio modulation," in Proc. IEEE Radio and wireless Conference (RAWCON'98), pp. 67-70, Colorado, Colo, USA, August 1998.

[3] F. Ramirez-Mireles and R.A. Scholtz, "Multiple-access performance limits with time hopping and pulse position modulation modulation," in Proc. IEEE Military Communications Conference (MILCOM'93), vol. 2, pp. 529-533, Boston, Mass, USA, Oct. 1993

[4] M.Z. Win and R.A. Scholtz, "Ultra-wide bandwidth time-hopping spreadspectrum impulse radio for wireless multiple access communications," IEEE Trans.Commun., vol. 48, no. 4, pp. 679-689, 2000.

[5] L.L.Yang and L. Hanzo, "Residue number system assisted fast frequency hopped synchronous ultra-wideband spread-spectrum multiple access: a design alternative to impulse radio," in IEEE J.Select. Areas Commun., vol 20. no. 9, pp. 1652-1663 Dec. 2002.

[6] J.R. Foerster, "The performance of direct-sequence spread ultra-wideband system in the presence of multipath, narrowband interference," in Proc. IEEE Conf. UWB Syst. Technol. (UWBST), vol 3, Baltimore, USA, May 2002, pp. 87-91. Colo, USA, August 1998.

[7] C.M. Canadeo, M.A. Temple, R.O. Baldwin, and R.A.Raines, "Code selection for enhancing UWB multiple access communication performance using TH-PPM and DS-BPSK modulation," in Proc. IEEE wireless Commun. Networking Conf. (WCNC), New orleans, LU, Mar. 2003.

[8] Nathaniel J.August, Rajesh Thirugnanam, and Dong Sam Ha, "An adaptive UWB modulation Scheme for optimization of energy, BER, and data rate," in International workshop on UWBST and IWUBS, pp. 182-186, May 2004.

[9] F.Ramirez-Mireles and R.A.Scholtz, "Multiple access with time hopping and block waveform PPM modulation," in Proc. IEEE International conference on Communications (ICC'98), vol. 2, pp. 775-779, Atlanta, Ga, USA, June 1998.

[10] G. Durisi and G. Romano, "On the validity of Gaussian approximation to characterize the multiuser capacity of UWB TH PPM," in Proc. IEEE Conference on Ultra Wideband Systems and Technologies (UWBST'02), vol. 1, pp. 157-161, Baltimore, Md, USA, May 2002.

[11] J.G. Proakis, Digital Communications, McGraw-Hill, New York, NY, USA, 4th edition, 2001 